\title{
A Novel Portable Device For Gamma And Neutron Spectroscopy With Special Nuclear Material Identification
}

\author{
Massimo Morichi \\ CAEN SpA \\ Viareggio, Italy \\ m.morichi@caen.it
}

\author{
Erica Fanchini \\ CAEN SpA \\ Viareggio, Italy \\ e.fanchini@caen.it
}

\author{
Giacomo Mangiagalli \\ CAEN SpA \\ Viareggio, Italy \\ g.mangiagalli@caen.it
}

\begin{abstract}
The development of new types of detectors and the increased performance of the electronics have paved the way for developing advanced systems for the measurement and identification of radioactive material that can be involved, for example, in illicit trafficking. Radioactive isotope identifiers are today commercially available. Nowadays those systems make use of inorganic scintillators as Sodium Iodide (NaI(TI)) or, for enhanced resolution, Lanthanum Bromide $\left(\mathrm{LaBr}_{3}\right)$ to identify the gamma emitters through their characteristic gamma lines. The most complete systems usually include an additional ${ }^{3} \mathrm{He}$ proportional counter for neutron detection and counting. The performances of such devices are compliant with standards as the IEC 62327 Hand Held Instruments for the Detection and Identification of Radionuclides. This paper presents a new type of portable radioactive isotope identifier. This device, based on an organic liquid scintillator with excellent Pulse Shape Discrimination (PSD) proprieties for the simultaneous detection of gamma rays and neutrons, detects radioactive source as Special Nuclear Material (SNM), medical, industrial and Naturally Occurring Radioactive Material. The exclusive feature of this instrument is the identification of neutron sources with discrimination between fission sources (like Californium

${ }^{252} \mathrm{Cf}$ ) and alpha-n type sources (like Americium Beryllium AmBe) from Plutonium and Uranium through an innovative dedicated algorithm. Individual thresholds for neutron and gamma counts are calculated to allow detection with $95 \%$ detection probability for a dose rate on the front face of the scintillator of at least $50 \mathrm{nSv} / \mathrm{h}$. Alarms are triggered separately when the respective rate exceed these thresholds. The neutron source detection has also been proved in a gamma ray field up to $100 \mu \mathrm{Sv} / \mathrm{h}$. The electronics is equipped with two analog inputs and two high voltage power supplies in a small form factor thus becoming an enabling technology for higher performance yet portable radioactive isotope identifier devices, which can include more detectors and perform data fusion analysis. The addition of a second detector allows to detect a masked neutron source through the PSD algorithm performed by the liquid scintillator detector while the added inorganic scintillator identifies the masking gamma emitters. The inorganic scintillator allows also the calculation of $P u$ and $U$ enrichment grade through characteristic gamma emission line.
\end{abstract}

Keywords-Special Nuclear Material (SNM), Uranium, Plutonium, neutron spectroscopy, homeland security

\section{INTRODUCTION}

The development of new types of detectors and the increased performance of the electronics have paved the way for developing advanced systems for the measurement and identification of radioactive material that can be involved, for example, in illicit trafficking. Radioactive isotope identifiers are today commercially available. Nowadays those systems make use of inorganic scintillators as Sodium Iodide NaI(Tl) or, for enhanced resolution, Lanthanum Bromide $\mathrm{LaBr} 3$ to identify the gamma emitters through their characteristic gamma lines. The most complete systems usually include an additional ${ }^{3} \mathrm{He}$ proportional counter for neutron detection and counting. The performances of such devices are compliant with standards as the IEC 62327 Hand Held Instruments for the Detection and Identification of Radionuclides.

\section{PSD ALGORITHM FOR SNM IDENTIFICATION}

This paper presents a new type of portable radioactive isotope identifier. This device, based on an organic liquid scintillator with excellent Pulse Shape Discrimination (PSD) proprieties for the simultaneous detection of gamma rays and neutrons, is able to detect radioactive source as Special Nuclear Material (SNM), medical, industrial and Naturally Occurring Radioactive Material (NORM). The main ability of this instrument is not limited to the detection, but also includes the identification of neutron sources with discrimination between fission sources (like 252Cf) and alpha-n type sources (like $\mathrm{Am} / \mathrm{Be}$ ) from Plutonium and Uranium through an innovative dedicated algorithm.

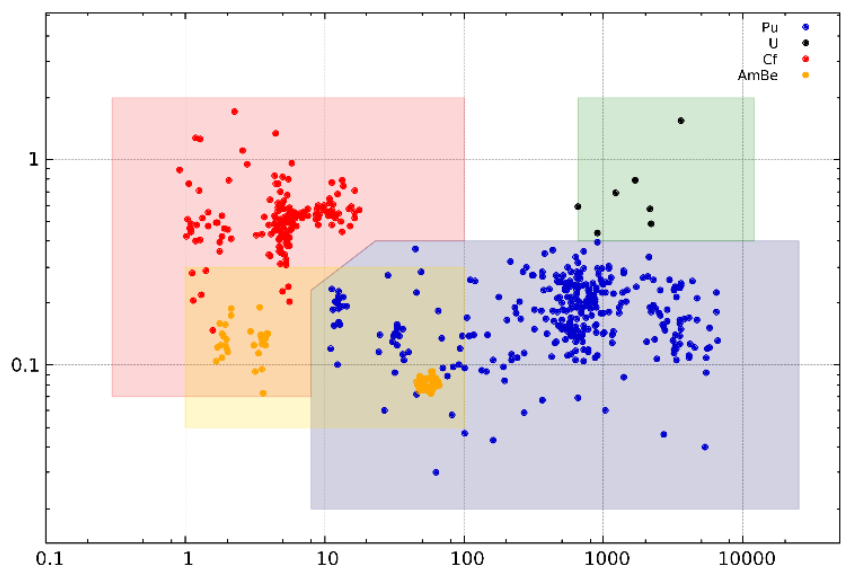

Fig. 1. Neutron source identification algorithm results. Experimental points for Cf and Pu correspond to: naked source, Poly shielded source and Lead shield source. The parameters used in the patented algorithm are plotted on $x$ and $y$ axis. Each point corresponds to the results obtained by the algorithm run on a spectrum collected in 1 min of identification. Each point can fall in the colored boxes which correspond to neutron sources present in the library. For Am-Be sources a third parameter is used in the algorithm

A moving average is used to integrate the last three minutes of natural background in order to set individual thresholds for neutron and gamma counts. Alarms are triggered separately when the respective rate exceed the alarm thresholds. 


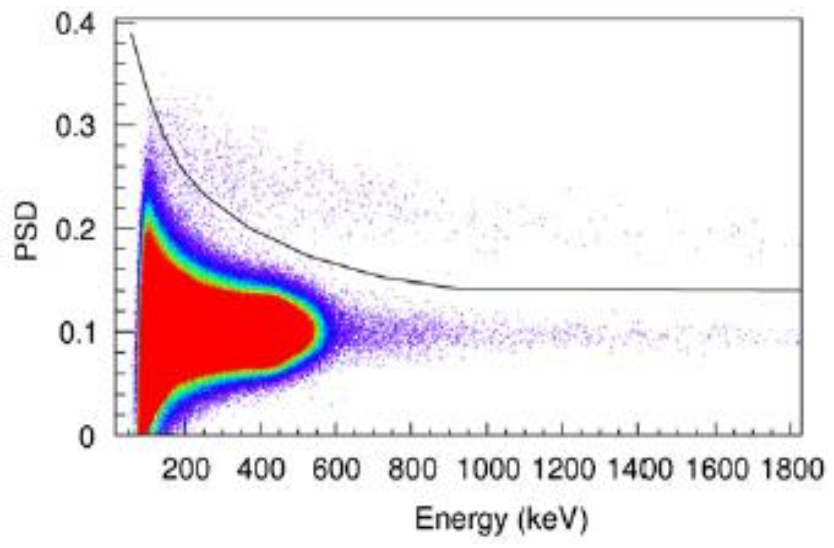

Fig. 2. Neutron-Gamma discrimination with gamma dose rate of $0.1 \mathrm{mSv} / \mathrm{h}$ on the front face of the detector. The black line is a polynomial function used to separate the gamma region from the neutron region. Pulse Shape Discrimination Plot obtained by a CAEN desktop digitizer with a EJ309 liquid scintillator detector coupled to a flat panel Hamamatsu.

These thresholds are calculated to allow detection with $95 \%$ detection probability at $95 \%$ confidence level for a dose rate on the front face of the scintillator of at least $50 \mathrm{nSv} / \mathrm{h}$. The neutron source detection has been tested also in high gamma ray fields up to $0.1 \mathrm{mSv} / \mathrm{h}$. The liquid scintillator detector is able to discriminate neutrons from gamma rays by using the PSD technique. Such signal processing is currently performed by using complex and expensive read-out systems. Today, thanks to the development of compact and fully digital pulse analyzers, it is possible to design compact systems with enhanced performance. The system here presented makes use of advanced digital electronics including a Multi Channel Analyzer (MCA) and a FPGA running Digital Pulse Processing (DPP) firmware for PSD. This firmware allows for online fast discrimination of gamma rays from neutrons thus enabling individual alarms for each kind of particle. The PSD firmware is based on an advanced online digital dual gate charge integration technique able to sustain high counting rates.

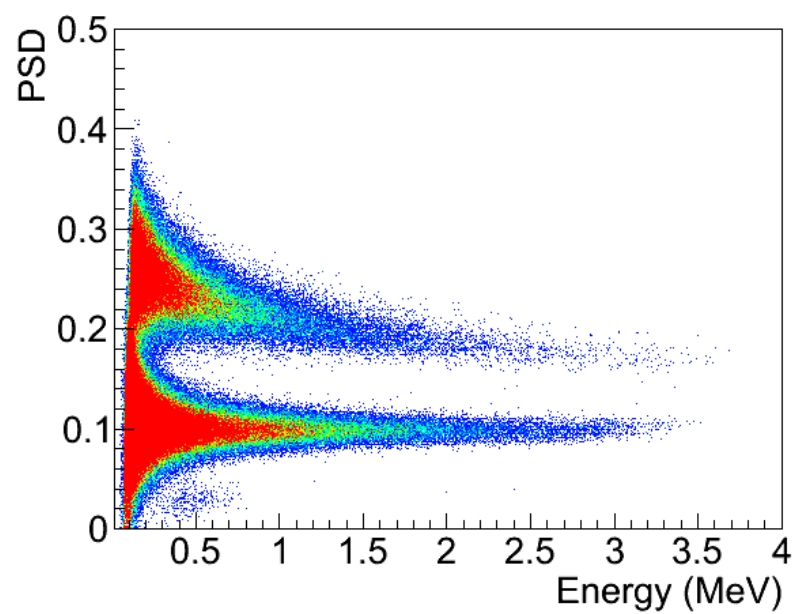

Fig. 3. Pulse Shape Discrimination Plot obtained by a CAEN desktop digitizer with a EJ309 liquid scintillator detector coupled to a flat panel Hamamatsu. The gamma region is between $0.0<P S D<0.2$ and the neutron region is between $0.2<P S D<0.4 . P S D=$ (long gate integration - short gate integration)/ long gate integration.

It performs input signal baseline calculation, self-triggering, double integration of both prompt and total charge for PSD and pedestal subtraction for energy calculation and pile-up rejection.

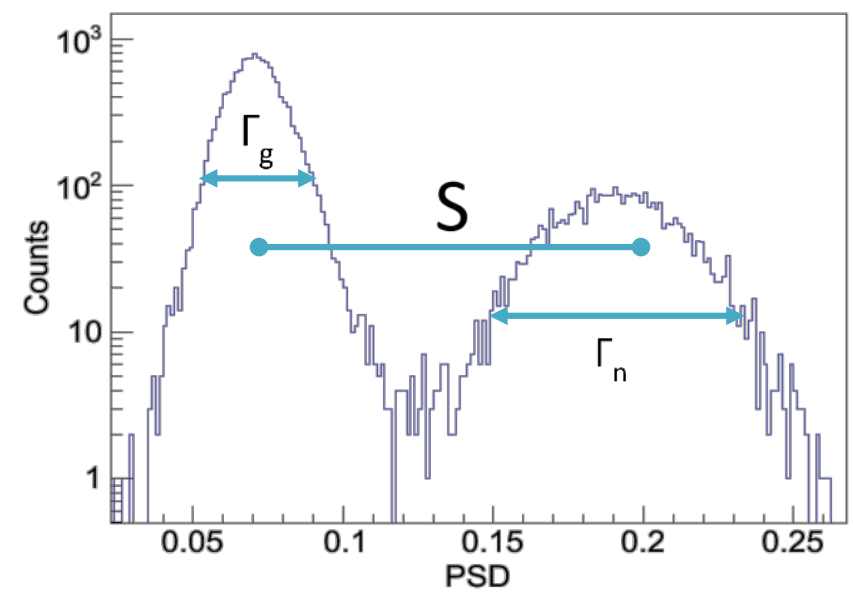

Fig.4. Typical example of FoM plot obtained by the projection of the PSD plot on the $x$ axis. FWHMs and separation between centroids are shown in the plot. Centroids and FWHM are obtained by Gaussian fit of the plot. The fit is not shown

Moreover, with a novel discrimination algorithm, a neutron source classification is also possible. An index of the performance of the Pulse Shape Discrimination is the Figure of Merit $(F O M)$. The graph for the FoM is obtained by projecting the $\mathrm{y}$ axis of the PSD plot on the $\mathrm{x}$ axis in a PSD vs count plot thus obtaining two Gaussian distribution, one for the gamma and one for the neutron (see Fig. 4). The FoM is defined as:

$$
\mathrm{FOM}=\mathrm{S} /\left(\Gamma_{\mathrm{n}}+\Gamma_{\mathrm{g}}\right)
$$

where $\mathrm{S}$ is the distance between the two centroids of neutron and gamma gaussian distribution and $\left(\Gamma_{\mathrm{n}}+\Gamma_{\mathrm{g}}\right)$ is the sum of gamma and neutron FWHMs [1]. The FoM obtained with the configuration described above in the caption of Fig. 3 and with an energy window of $480 \pm 75 \mathrm{keV}$ is 1.73 .

\section{NEUTRON SOURCE IDENTIFICATION ALGORITHM}

The results described in the above section were obtained with the digitizer and the detectors arranged in a laboratory set-up. The algorithm was run with ad-hoc program run on a desktop pc. To target the engineering of this set up, a more complicated scenario was set up.

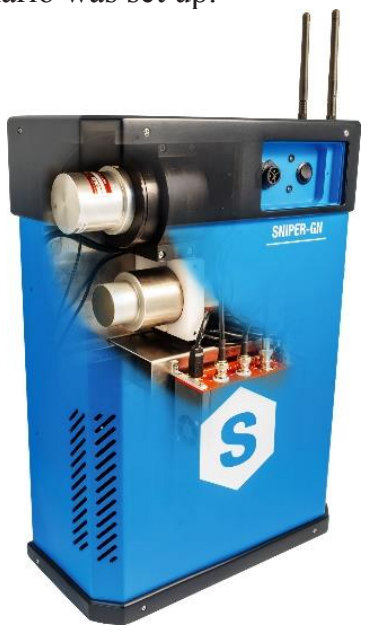

Fig. 5. Aluminum box set-up containing the two detectors, the DT5790, the $\mathrm{LiFePO}_{4}$ Battery and the Single Board Computer (SBC). The data are transmitted through $\mathrm{Wi}-\mathrm{Fi}$. 
The system is packaged in an aluminum box containing the digitizer and the detectors. It is powered with a $\mathrm{LiFePO}_{4}$ battery with capacity of $25 \mathrm{Ah}$ at $12 \mathrm{~V}$. The computer is replaced with Single Board Computer (SBC) equipped with ARM processor which run the optimized algorithms. In this configuration the system becomes portable. Some homeland security standards were identified to better understand where this kind of set up can be included. There isn't a specific standard for this kind of device but the most suitable are:

- IEC 62327 Hand Held instruments for the detection and identification of radionuclides

- ANSI 42.34 Hand Held instruments for the detection and identification of radionuclides

Considering the European standard the requirements for gamma and neutron alarm and identification are:

- Gamma alarm raised in 1 second for a source moving at a speed of $0.5 \mathrm{~m} / \mathrm{s}$ producing a radiation field of $500 \mathrm{nSv} / \mathrm{h}$ above the bakground at the reference point of the RID at a closest distance of 1 m.

- Neutron alarm raised in 2 seconds for a Cf-252 source having an emission rate of 20.000 neutrons/s surrounded by $4 \mathrm{~cm}$ of High-density poliethilene at $25 \mathrm{~cm}$ of distance from the reference point of the RID

- 1 minute or less for source identification

Tests were repeated with the new and more complicated setup. Most of the measurement were focused on the neutron alarm and the neutron source identification performed by the patented algorithm (see Fig. 1). The identification measurement is performed on a 1 minute spectra as set as maximum in the standard. Bigger distance respect to the standard were also tested. The Legnaro laboratories use a stronger Cf-252 respect to the standard, so the distance of 25 $\mathrm{cm}$ was scaled proportionally and the new reference standard distance is set to $45.5 \mathrm{~cm}$.

Many test were performed with different thickness of lead, poliethilene and mixed shields.

Table I. Cf-252 source identification test performed with EJ309 and DT5790 packaged in a aluminum box and powered by a $\mathrm{LiFePO}_{4}$ battery. Identification patented algorithm is run by a SBC computer with ARM processor

\begin{tabular}{ccc}
\hline distance (cm) & Shielding & $\begin{array}{c}\text { Success/ } \\
\text { trials }\end{array}$ \\
\hline standard & Pb $5 \mathrm{~cm}+$ Poly $6 \mathrm{~cm}$ & $10 / 10$ \\
2 x standard & Poly $10 \mathrm{~cm}$ & $10 / 10$ \\
4.5 x standard & - & $15 / 15$ \\
\hline
\end{tabular}

Other tests were performed with different neutron source by scaling the neutron emission rate proportionally to standard definition. The best result is obtained with a special mixed source enriched in $\mathrm{Pu}-239$ at $61 \%$. With this source a $100 \%$ success of identification ( 30 success on 30 trials) is obtained at 23 times the standard distance.

\section{DOUBLE CHANNEL ELECTRONIC FOR GAMMA RADIATION DETECTOR}

The electronics is equipped with two analog inputs and two high voltage power supplies in a very small form factor thus becoming an enabling technology for higher performance yet portable radioactive isotope identifier devices, which can include more detectors and perform data fusion analysis. The double channel capabilities of the electronics are exploited by adding another detector to the system for improving the identification performance of the gamma emitters. A highresolution inorganic scintillator, such as $\mathrm{LaBr}_{3}$ or $\mathrm{CeBr}_{3}$, has been easily integrated into the system to perform an enhanced gamma radioisotope identification by characteristic gamma lines recognition. This solution allows for a simultaneous identification of multiple radionuclides, exceeding therefore the limits of gamma identification performed with organic liquid scintillator and Compton spectra libraries only. The added benefit of this solution is the possibility to detect a masked neutron source through the PSD algorithm performed by the liquid scintillator detector while the inorganic scintillator identifies the masking gamma emitters. The inorganic scintillator enhances also the neutron source identification. In fact, thanks to the high resolution of the crystal, it is possible to calculate the $\mathrm{Pu}$ and $\mathrm{U}$ enrichment grade through their characteristic gamma emission rate ratio as well.
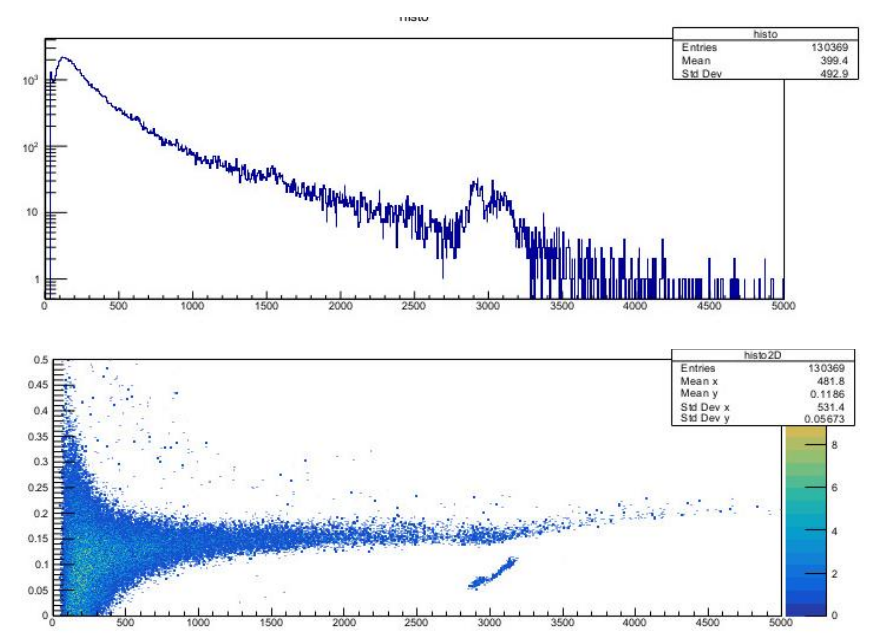

Fig. 6. Pulse Shape Discrimination Plot obtained by a CAEN desktop digitizer with a NaIL Tм 2 "x2" coupled to a standard PMT. The thermal neutron region is the spot around $3000 \mathrm{keV} . P S D=1$ - (short gate integration/ long gate integration). For this detector the neutron region is below the gamma region because the Lithium dopant make the gamma signal slower

\section{INNOVATIVE NAI(TL) FOR THERMAL NEUTRON DETECTION}

An innovative feature is under development. Some tests are running to study the performance of an innovative detector consisting of $\mathrm{NaI}(\mathrm{Tl})$ detector doped with Lithium. This detector unifies the gamma scintillation proprieties of a $\mathrm{NaI}(\mathrm{Tl})$ detector with the thermal neutron detection capabilities. NaIL ${ }^{\mathrm{TM}}$ detector is provided by Saint-Gobain and with this detector neutrons and gamma rays are easily distinguished through pulse shape discrimination (PSD). With the addition of Li into the NaI matrix, gamma ray scintillation pulses become longer than neutron reaction pulses. The effect is dramatic, and simple PSD techniques create complete separation [2]. Some test has been performed with a 2"x2" NaIL $^{\text {TM }}$ detector coupled with a standard PMT tube and analysed with a CAEN DT5725 digitizer (14-bit@250 MS/s) with the DPP-PSD firmware. With a dynamic range of 0.5 $\mathrm{Vpp}$ and a long gate integration of $4000 \mathrm{~ns}$ the best resolution obtained at $662 \mathrm{keV}$ with a Cs-137 source is $6.6 \%$ which is comparable with standard $\mathrm{NaI}(\mathrm{Tl})$ detector of the same 
dimension. The detector was also tested with a Cf-252, a source of fast neutrons and gamma rays, placed at $30 \mathrm{~cm}$ and shielded with different thickness of polyethylene $(0,2,4$ and $6 \mathrm{~cm}$ ) adjacent to the source. Using the Pulse Shape Discrimination, the signals produced in the detector by the interaction of polyethylene slowed down neutron were identified. The 2-D plot of PSD vs energy is shown is Fig. 6. Around the $3 \mathrm{MeV}$ energy the contribution of thermal neutron can be noted. This contribution is produced by thermal neutrons that release energy inside the detector by interacting with Lithium according to the reaction:

$$
\mathrm{n}+{ }^{6} \mathrm{Li} \rightarrow{ }^{3} \mathrm{H}+{ }^{4} \mathrm{He}
$$

The maximum thermal neutron efficiency is obtained in the configuration with $6 \mathrm{~cm}$ of polyethylene and the neutron efficiency obtained for NaIL TM is the $89 \%$ with respect to the EJ-420 detector, with a diameter of 2" and placed in the same geometrical configuration and with the same activity of the source. The EJ-420 can be considered as a standard for the measurement of thermal neutrons. Replacing the $\mathrm{CeBr}_{3}$ with this innovative detector can add a lot of benefits to the device. First of all, the system with two detectors can detect and identify gamma, thermal neutron and fast neutron sources. Further information on the polyethylene shielding thickness can be retrieved by the thermal to fast neutron ratio. The only deterioration is the worse resolution of a $\mathrm{NaI}(\mathrm{Tl})$ compared to the $\mathrm{CeBr}_{3}$. This feature don't affect the gamma identification algorithm dramatically; in fact most of the Radiation Isotope Identification Device is equipped with a $\mathrm{NaI}(\mathrm{Tl})$ detector and can easily identify gamma radiation source.

\section{REFERENCES}

[1] J.R.M Annand, Nuclear Instruments and Methods A 262 (1987) 371.

[2] K. Yang, P. R. Merge at al, a large volume neutron-gamma scintillator with exceptional pulse shape discrimination, IEEE NSS-MIC. 